\title{
AN INVOLUTION BASED LEFT IDEAL IN THE HECKE ALGEBRA
}

\author{
G. LUSZTIG
}

Abstract. We show that the Hecke algebra module carried by the involutions in a Weyl group (defined by the author and Vogan) can be identified with a left ideal in the Hecke algebra. An analogous result is proved for any Coxeter group.

\section{INTRODUCTION}

0.1. Let $W$ be a Coxeter group with set of simple reflections $S$ and with length function $l: W \rightarrow \mathbf{N}$. Let $u$ be an indeterminate. Let $\mathfrak{H}$ be the $\mathbf{Q}(u)$-vector space with basis $\left\{T_{w} ; w \in W\right\}$. We regard $\mathfrak{H}$ as an associative $\mathbf{Q}(u)$-algebra (with 1 ) with multiplication defined by $T_{w} T_{w^{\prime}}=T_{w w^{\prime}}$ if $l\left(w w^{\prime}\right)=l(w)+l\left(w^{\prime}\right),\left(T_{s}+1\right)\left(T_{s}-u^{2}\right)=$ 0 if $s \in S$. Let $*: W \rightarrow W\left(\right.$ or $w \mapsto w^{*}$ ) be an automorphism of $W$ such that $S^{*}=S, *^{2}=1$. Let $\mathbf{I}_{*}=\left\{w \in W ; w^{*}=w^{-1}\right\}$ be the set of "twisted involutions" of $W$. Let $M$ be the $\mathbf{Q}(u)$-vector space with basis $\left\{a_{w} ; w \in \mathbf{I}_{*}\right\}$. Following [LV], for any $s \in S$ we define a $\mathbf{Q}(u)$-linear map $T_{s}: M \rightarrow M$ by

$$
\begin{aligned}
& T_{s} a_{w}=u a_{w}+(u+1) a_{s w} \text { if } s w=w s^{*}>w ; \\
& T_{s} a_{w}=\left(u^{2}-u-1\right) a_{w}+\left(u^{2}-u\right) a_{s w} \text { if } s w=w s^{*}<w ; \\
& T_{s} a_{w}=a_{s w s^{*}} \text { if } s w \neq w s^{*}>w ; \\
& T_{s} a_{w}=\left(u^{2}-1\right) a_{w}+u^{2} a_{s w s^{*}} \text { if } s w \neq w s^{*}<w .
\end{aligned}
$$

(For $x, y$ in $W$ such that $y^{-1} x \in S$ or $x y^{-1} \in S$ we write $x<y$ or $y>x$ instead of $l(x)=l(y)-1$.) According to [LV] and [L5], these linear maps define an $\mathfrak{H}$-module structure on $M$. Let $\hat{\mathfrak{H}}$ be the vector space consisting of all formal (possibly infinite) sums $\sum_{x \in W} c_{x} T_{x}$ where $c_{x} \in \mathbf{Q}(u)$. We can view $\mathfrak{H}$ as a subspace of $\hat{\mathfrak{H}}$ in an obvious way. The $\mathfrak{H}$-module structure on $\mathfrak{H}$ (left multiplication) extends in an obvious way to an $\mathfrak{H}$-module structure on $\hat{\mathfrak{H}}$. We set

$$
X=\sum_{x \in W ; x^{*}=x} u^{-l(x)} T_{x} \in \hat{\mathfrak{H}} .
$$

The following is the main result of this paper:

Theorem 0.2. (a) There exists a unique $\mathfrak{H}$-linear map $\mu: M \rightarrow \hat{\mathfrak{H}}$ such that $\mu\left(a_{1}\right)=X$. Moreover, $\mu$ is an isomorphism of $M$ onto the $\mathfrak{H}$-submodule of $\hat{\mathfrak{H}}$ generated by $X$.

(b) Let $z \in \mathbf{I}_{*}$; we set $\mu\left(a_{z}\right)=\sum_{x \in W} N_{z}^{x} T_{x}$ where $N_{z}^{x} \in \mathbf{Q}(u)$. For any $x \in W$ we have $N_{z}^{x} \in \mathbf{Z}\left[u^{-1}\right]$, hence we can define $n_{z}^{x}=\left.N_{z}^{x}\right|_{u^{-1}=0} \in \mathbf{Z}$.

Received by the editors February 8, 2016.

2010 Mathematics Subject Classification. Primary 20G99.

The author was supported in part by National Science Foundation grant 1303060 . 
(c) There is a unique surjective function $\pi: W \rightarrow \mathbf{I}_{*}$ such that for $x \in W, z \in \mathbf{I}_{*}$ we have $n_{z}^{x}=1$ if $z=\pi(x), n_{z}^{x}=0$ if $z \neq \pi(x)$. (Note that $\pi(1)=1$.)

This was conjectured in [L4, 3.4, 3.7] where it was verified for several $W$ of low rank. In the case where $W$ is of type $A_{n}$ and $*=1$, part (a) of the theorem was first proved by $\mathrm{Hu}$ and Zhang [HZ. The proof of the theorem is given in Section 1. In Section 2 we will discuss a special case of Theorem 0.2. Section 3 is preparatory for Section 4. In Section 4 we discuss some applications of Theorem 0.2 . For example, we show that if $W$ is a Weyl group of type $A_{n}$ and if $E$ is an irreducible representation of $\mathfrak{H}$, then the action of $X$ on $E$ is through an operator of rank 1; in particular, the image of this operator is a canonical line in $E$. As another application we show that if $W$ is a Weyl group of classical type and $E$ is an irreducible special representation of the asymptotic Hecke algebra attached to $W$ then $E$ admits a basis such that any canonical basis element of that algebra acts in this basis through a matrix with all entries in $\mathbf{N}$.

A third application is a definition of a canonical $G\left(\mathbf{F}_{q}\right)$-stable subpace $\mathcal{F}^{\prime}$ of the space of functions $\mathcal{F}$ on the flag manifold of a Chevalley group $G\left(\mathbf{F}_{q}\right)$ over a finite field $\mathbf{F}_{q}$ with the following properties: if $G=S L_{n}$, then $\mathcal{F}^{\prime}$ contains exactly one copy of each irreducible representation of $G\left(\mathbf{F}_{q}\right)$ which appears in $\mathcal{F}$; in general, the dimension of $\mathcal{F}^{\prime}$ is a polynomial in $q$ with coefficients in $\mathbf{N}$ whose value at 1 is the number of involutions in $W$. This polynomial is the sum of the fake degrees of the various irreducible representations of the Hecke algebra (each one taken once).

\section{Proof of Theorem 0.2}

1.1. The $\mathbf{Z}[u]$-submodule of $M$ with basis $\left\{a_{w} ; w \in \mathbf{I}_{*}\right\}$ is stable under the maps $T_{s}: M \rightarrow M(s \in S)$ hence is stable under the action of $T_{x}(x \in W)$ since $T_{x}$ is a composition of various $T_{s}$. Hence for $x \in W$ we can write uniquely

$$
T_{x} a_{1}=\sum_{z \in \mathbf{I}_{*}} L_{z}^{x} a_{z}
$$

where $L_{z}^{x} \in \mathbf{Z}[u]$.

1.2. For $x \in W, z \in \mathbf{I}_{*}, s \in S$ we show:

$$
\begin{aligned}
& \left(u^{2}-u\right) L_{s z}^{x}=u^{2} L_{z}^{s x}+\left(u^{2}-u-1\right) L_{z}^{x} \text { if } s z=z s^{*}>z, s x<x ; \\
& (u+1) L_{s z}^{x}-u L_{z}^{x}=u^{2} L_{z}^{s x} \text { if } s z=z s^{*}<z, s x<x \\
& u^{2} L_{s z s^{*}}^{x}=u^{2} L_{z}^{s x}+\left(u^{2}-1\right) L_{z}^{x} \text { if } s z \neq z s^{*}>z, s x<x \\
& L_{s z s^{*}}^{x}=u^{2} L_{z}^{s x} \text { if } s z \neq z s^{*}<z, s x<x \\
& u L_{z}^{x}+\left(u^{2}-u\right) L_{s z}^{x}=L_{z}^{s x} \text { if } s z=z s^{*}>z, s x>x \\
& (u+1) L_{s z}^{x}+\left(u^{2}-u-1\right) L_{z}^{x}=L_{z}^{s x} \text { if } s z=z s^{*}<z, s x>x ; \\
& u^{2} L_{s z s^{*}}^{x}=L_{z}^{s x} \text { if } s z \neq z s^{*}>z, s x>x \\
& L_{s z s^{*}}^{x}+\left(u^{2}-1\right) L_{z}^{x}=L_{z}^{s x} \text { if } s z \neq z s^{*}<z, s x>x .
\end{aligned}
$$

1.3. For $x \in W, s \in S$ we have $T_{s} T_{x} a_{1}=\sum_{z \in \mathbf{I}_{*}} L_{z}^{x} T_{s} a_{z}$. Note that $T_{s} T_{x} a_{1}=T_{s x} a_{1}$ if $s x>x$ and $T_{s} T_{x} a_{1}=u^{2} T_{s x} a_{1}+\left(u^{2}-1\right) T_{x} a_{1}$ if $s x<x$. Thus,

$$
\begin{aligned}
\sum_{z \in \mathbf{I}_{*}} L_{z}^{x} T_{s} a_{z} & =\sum_{z \in \mathbf{I}_{*}} L_{z}^{s x} a_{z} \text { if } s x>x \\
\sum_{z \in \mathbf{I}_{*}} L_{z}^{x} T_{s} a_{z} & =\sum_{z \in \mathbf{I}_{*}} u^{2} L_{z}^{s x} a_{z}+\sum_{z \in \mathbf{I}_{*}}\left(u^{2}-1\right) L_{z}^{x} a_{z} \text { if } s x<x .
\end{aligned}
$$


Using the formulas for $T_{s} a_{z}$ in 0.1 we see that

$$
\begin{aligned}
& \quad \sum_{z \in \mathbf{I}_{*} ; s z=z s^{*}, s z>z} L_{z}^{x}\left(u a_{z}+(u+1) a_{s z}\right) \\
& +\sum_{z \in \mathbf{I}_{*} ; s z=z s^{*}, s z<z} L_{z}^{x}\left(\left(u^{2}-u-1\right) a_{z}+\left(u^{2}-u\right) a_{s z}\right) \\
& +\sum_{z \in \mathbf{I}_{*} ; s z \neq z s^{*}, s z>z} L_{z}^{x} a_{s z s^{*}}+\sum_{z \in \mathbf{I}_{*} ; s z \neq z s^{*}, s z<z} L_{z}^{x}\left(\left(u^{2}-1\right) a_{z}+u^{2} a_{s z s^{*}}\right)
\end{aligned}
$$

or, equivalently,

$$
\begin{aligned}
& \sum_{z \in \mathbf{I}_{*} ; s z=z s^{*}, s z>z} u L_{z}^{x} a_{z}+\sum_{z \in \mathbf{I}_{*} ; s z=z s^{*}, s z<z}(u+1) L_{s z}^{x} a_{z} \\
& +\sum_{z \in \mathbf{I}_{*} ; s z=z s^{*}, s z<z}\left(u^{2}-u-1\right) L_{z}^{x} a_{z}+\sum_{z \in \mathbf{I}_{*} ; s z=z s^{*}, s z>z}\left(u^{2}-u\right) L_{s z}^{x} a_{z} \\
& +\sum_{z \in \mathbf{I}_{*} ; s z \neq z s^{*}, s z<z} L_{s z s^{*}}^{x} a_{z}+\sum_{z \in \mathbf{I}_{*} ; s z \neq z s^{*}, s z<z}\left(u^{2}-1\right) L_{z}^{x} a_{z} \\
& +\sum_{z \in \mathbf{I}_{*} ; s z \neq z s^{*}, s z>z} u^{2} L_{s z s^{*}}^{x} a_{z}
\end{aligned}
$$

is equal to

$$
\sum_{z \in \mathbf{I}_{*}} L_{z}^{s x} a_{z} \text { if } s x>x
$$

or to

$$
\sum_{z \in \mathbf{I}_{*}} u^{2} L_{z}^{s x} a_{z}+\sum_{z \in \mathbf{I}_{*}}\left(u^{2}-1\right) L_{z}^{x} a_{z} \text { if } s x<x .
$$

We now take the coefficients of $a_{z}$ in the two sides of this equality. We obtain the equalities in 1.2 .

1.4. Let ${ }^{-}: \mathbf{Z}\left[u, u^{-1}\right] \rightarrow \mathbf{Z}\left[u, u^{-1}\right]$ be the ring involution such that $\overline{u^{n}}=(-u)^{-n}$ for any $n \in \mathbf{Z}$. We apply ${ }^{-}$to the equalities in 1.2 and multiply the resulting equalities by $u^{2}$. We obtain the following equalities:

$$
\begin{aligned}
& (1+u) \bar{L}_{s z}^{x}=\bar{L}_{z}^{s x}+\left(-u^{2}+u+1\right) \bar{L}_{z}^{x} \text { if } s z=z s^{*}>z, s x<x ; \\
& \left(u^{2}-u\right) \bar{L}_{s z}^{x}+u \bar{L}_{z}^{x}=\bar{L}_{z}^{s x} \text { if } s z=z s^{*}<z, s x<x ; \\
& \bar{L}_{s z s^{*}}^{x}=\bar{L}_{z}^{s x}+\left(1-u^{2}\right) \bar{L}_{z}^{x} \text { if } s z \neq z s^{*}>z, s x<x ; \\
& u^{2} \bar{L}_{s z s^{*}}=\bar{L}_{z}^{s x} \text { if } s z \neq z s^{*}<z, s x<x ; \\
& -u \bar{L}_{z}^{x}+(1+u) \bar{L}_{s z}^{x}=u^{2} \bar{L}_{z}^{s x} \text { if } s z=z s^{*}>z, s x>x ; \\
& \left(u^{2}-u\right) \bar{L}_{s z}^{x}+\left(-u^{2}+u+1\right) \bar{L}_{z}^{x}=u^{2} \bar{L}_{z}^{s x} \text { if } s z=z s^{*}<z, s x>x ; \\
& \bar{L}_{s z s^{*}}^{x}=u^{2} \bar{L}_{z}^{s x} \text { if } s z \neq z s^{*}>z, s x>x ; \\
& u^{2} \bar{L}_{s z s^{*}}+\left(1-u^{2}\right) \bar{L}_{z}^{x}=u^{2} \bar{L}_{z}^{s x} \text { if } s z \neq z s^{*}<z, s x>x .
\end{aligned}
$$

1.5. It is well known (see for example [L5]) that there is a unique function $\phi: \mathbf{I}_{*} \rightarrow$ $\mathbf{N}$ such that $\phi(1)=0$ and such that for any $z \in \mathbf{I}_{*}$ and any $s \in S$ such that $s z<z$ we have $\phi(z)=\phi(s z)+1$ if $s z=z s^{*}$ and $\phi(z)=\phi\left(s z s^{*}\right)$ if $s z \neq z s^{*}$. By induction on $l(z)$ we see that $\phi(z)=l(z) \bmod 2$ for any $z \in \mathbf{I}_{*}$. Hence for $z \in \mathbf{I}_{*}$ we can set $\epsilon(z)=(-1)^{(l(z)+\phi(z)) / 2}$. From the definitions we see that for any $z \in \mathbf{I}_{*}$ and any $s \in S$ we have
(a) $\epsilon(z)=-\epsilon(s z)$ if $s z=z s^{*}$ and $\epsilon(z)=-\epsilon\left(s z s^{*}\right)$ if $s z \neq z s^{*}$. 
1.6. For $x \in W, z \in \mathbf{I}_{*}$ we set

$$
\tilde{L}_{z}^{x}=(-1)^{l(x)} \epsilon(z) \bar{L}_{z}^{x} .
$$

With this notation the formulas in 1.4 can be rewitten as follows:

$$
\begin{aligned}
& (1+u) \tilde{L}_{s z}^{x}=\tilde{L}_{z}^{s x}+\left(u^{2}-u-1\right) \tilde{L}_{z}^{x} \text { if } s z=z s^{*}>z, s x<x ; \\
& \left(u^{2}-u\right) \tilde{L}_{s z}^{x}-u \tilde{L}_{z}^{x}=\tilde{L}_{z}^{s x} \text { if } s z=z s^{*}<z, s x<x ; \\
& \tilde{L}_{s z s^{*}}^{x}=\tilde{L}_{z}^{s x}+\left(u^{2}-1\right) \tilde{L}_{z}^{x} \text { if } s z \neq z s^{*}>z, s x<x ; \\
& u^{2} \tilde{L}_{s z s^{*}}^{x}=\tilde{L}_{z}^{s x} \text { if } s z \neq z s^{*}<z, s x<x ; \\
& u \tilde{L}_{z}^{x}+(1+u) \tilde{L}_{s z}^{x}=u^{2} \tilde{L}_{z}^{s x} \text { if } s z=z s^{*}>z, s x>x ; \\
& \left(u^{2}-u\right) \tilde{L}_{s z}^{x}+\left(u^{2}-u-1\right) \tilde{L}_{z}^{x}=u^{2} \tilde{L}_{z}^{s x} \text { if } s z=z s^{*}<z, s x>x ; \\
& \tilde{L}_{s z s^{*}}^{x}=u^{2} \tilde{L}_{z}^{s x} \text { if } s z \neq z s^{*}>z, s x>x ; \\
& u^{2} \tilde{L}_{s z s^{*}}^{x}+\left(u^{2}-1\right) \tilde{L}_{z}^{x}=u^{2} \tilde{L}_{z}^{s x} \text { if } s z \neq z s^{*}<z, s x>x .
\end{aligned}
$$

1.7. Giving an $\mathfrak{H}$-linear map $\mu: M \rightarrow \hat{\mathfrak{H}}$ is the same as giving a family of elements $Y_{z} \in \hat{\mathfrak{H}}$ (one for each $z \in \mathbf{I}_{*}$ ) such that for any $z \in \mathbf{I}_{*}, s \in S$ we have:

$$
\begin{aligned}
& T_{s} Y_{z}=u Y_{z}+(u+1) Y_{s z} \text { if } s z=z s^{*}>z ; \\
& T_{s} Y_{z}=\left(u^{2}-u-1\right) Y_{z}+\left(u^{2}-u\right) Y_{s z} \text { if } s z=z s^{*}<z ; \\
& T_{s} Y_{z}=Y_{s z s^{*}} \text { if } s z \neq z s^{*}>z ; \\
& T_{s} Y_{z}=\left(u^{2}-1\right) Y_{z}+u^{2} Y_{s z s^{*}} \text { if } s z \neq z s^{*}<z .
\end{aligned}
$$

Indeed, if $\mu$ is given then the elements $Y_{z}=\mu\left(a_{z}\right)$ satisfy the equations above. Conversely, if the elements $Y_{z}$ are given as above then we can define a $\mathbf{Q}(u)$-linear map $\mu: M \rightarrow \hat{\mathfrak{H}}$ by $\mu\left(a_{z}\right)=Y_{z}$ for all $z \in \mathbf{I}_{*}$. This map will be compatible with the action of $T_{s}$ for any $s \in S$ hence it will be automatically $\mathfrak{H}$-linear. Setting $Y_{z}=\sum_{x \in W} N_{z}^{x} T_{z}$ where $N_{z}^{x} \in \mathbf{Q}(u)$ we see that giving an $\mathfrak{H}$-linear map $\mu: M \rightarrow \hat{\mathfrak{H}}$ is the same as giving a family of elements $\left\{N_{z}^{x} ;(x, z) \in W \times \mathbf{I}_{*}\right\}$ in $\mathbf{Q}(u)$ such that the following equations are satisfied for any $z \in \mathbf{I}_{*}, s \in S$ :

$$
\begin{aligned}
& \sum_{x \in W} N_{z}^{x} T_{s} T_{x}=\sum_{x \in W} u N_{z}^{x} T_{x}+\sum_{x \in W}(u+1) N_{s z}^{x} T_{x} \text { if } s z=z s^{*}>z ; \\
& \sum_{x \in W} N_{z}^{x} T_{s} T_{x}=\sum_{x \in W}\left(u^{2}-u-1\right) N_{z}^{x} T_{x}+\sum_{x \in W}\left(u^{2}-u\right) N_{s z}^{x} T_{x} \text { if } s z=z s^{*}<z ; \\
& \sum_{x \in W} N_{z}^{x} T_{s} T_{x}=\sum_{x \in W} N_{s z s^{*}}^{x} T_{x} \text { if } s z \neq z s^{*}>z ; \\
& \sum_{x \in W} N_{z}^{x} T_{s} T_{x}=\sum_{x \in W}\left(u^{2}-1\right) N_{z}^{x} T_{x}+\sum_{x \in W} u^{2} N_{s z s^{*}}^{x} T_{x} \text { if } s z \neq z s^{*}<z .
\end{aligned}
$$

(We then say that the family $\left\{N_{z}^{x} ;(x, z) \in W \times \mathbf{I}_{*}\right\}$ is admissible.) Here we replace

$$
\begin{aligned}
& \sum_{x \in W} N_{z}^{x} T_{s} T_{x}=\sum_{x \in W ; s x>x} N_{z}^{x} T_{s x}+\sum_{x \in W ; s x<x} u^{2} N_{z}^{x} T_{s x}+\sum_{x \in W ; s x<x}\left(u^{2}-1\right) N_{z}^{x} T_{x} \\
& =\sum_{x \in W ; s x<x} N_{z}^{s x} T_{x}+\sum_{x \in W ; s x>x} u^{2} N_{z}^{s x} T_{x}+\sum_{x \in W ; s x<x}\left(u^{2}-1\right) N_{z}^{x} T_{x} \\
& =\sum_{x \in W ; s x<x}\left(N_{z}^{s x}+\left(u^{2}-1\right) N_{z}^{x}\right) T_{x}+\sum_{x \in W ; s x>x} u^{2} N_{z}^{s x} T_{x} .
\end{aligned}
$$

We see that the condition $\left\{N_{z}^{x} ;(x, z) \in W \times \mathbf{I}_{*}\right\}$ is admissible is equivalent to the following set of equations (with $x \in W, z \in \mathbf{I}_{*}, s \in S$ ):

$$
\begin{aligned}
& (1+u) N_{s z}^{x}=N_{z}^{s x}+\left(u^{2}-u-1\right) N_{z}^{x} \text { if } s z=z s^{*}>z, s x<x \\
& \left(u^{2}-u\right) N_{s z}^{x}-u N_{z}^{x}=N_{z}^{s x} \text { if } s z=z s^{*}<z, s x<x \\
& N_{s z s^{*}}^{x}=N_{z}^{s x}+\left(u^{2}-1\right) N_{z}^{x} \text { if } s z \neq z s^{*}>z, s x<x \\
& u^{2} N_{s z s^{*}}^{x}=N_{z}^{s x} \text { if } s z \neq z s^{*}<z, s x<x \\
& u N_{z}^{x}+(1+u) N_{s z}^{x}=u^{2} N_{z}^{s x} \text { if } s z=z s^{*}>z, s x>x
\end{aligned}
$$




$$
\begin{aligned}
& \left(u^{2}-u\right) N_{s z}^{x}+\left(u^{2}-u-1\right) N_{z}^{x}=u^{2} N_{z}^{s x} \text { if } s z=z s^{*}<z, s x>x \\
& N_{s z s^{*}}^{x}=u^{2} N_{z}^{s x} \text { if } s z \neq z s^{*}>z, s x>x \\
& u^{2} N_{s z s^{*}}^{x}+\left(u^{2}-1\right) N_{z}^{x}=u^{2} N_{z}^{s x} \text { if } s z \neq z s^{*}<z, s x>x
\end{aligned}
$$

Comparing with the formulas in 1.6 , we see that the family $\left\{\tilde{L}_{z}^{x} ;(x, z) \in W \times \mathbf{I}_{*}\right\}$ is admissible. Hence there is a unique $\mathfrak{H}$-linear map $\mu: M \rightarrow \hat{\mathfrak{H}}$ such that for any $z \in \mathbf{I}_{*}$ we have $\mu\left(a_{z}\right)=\sum_{x \in W} \tilde{L}_{z}^{x} T_{z}$. Since $L_{1}^{x}=\delta_{x, x^{*}} u^{l(x)}$ (see [L6, 1.8]), we have $\tilde{L}_{1}^{x}=\delta_{x, x^{*}}(-1)^{l(x)}(-u)^{-l(x)}=\delta_{x, x^{*}} u^{-l(x)}$, so that $\mu\left(a_{1}\right)=X$ (see 0.1). Thus the existence part in $0.2(\mathrm{a})$ is established. The uniqueness part in $0.2(\mathrm{a})$ is obvious since $a_{1}$ generates $M$ as an $\mathfrak{H}$-module. Since $L_{z}^{x} \in \mathbf{Z}[u]$ we see that $\tilde{L}_{z}^{x} \in \mathbf{Z}\left[u^{-1}\right]$ and $0.2(\mathrm{~b})$ is established.

1.8. The algebra $\mathfrak{H}$ and its module $M$ can be specialized to $u=0$. Then $\mathfrak{H}$ becomes a Q-algebra $\mathfrak{H}_{0}$ with basis $\left\{\underline{T}_{w} ; w \in W\right\}$ and multiplication given by $\underline{T}_{w} \underline{T}_{w^{\prime}}=\underline{T}_{w w^{\prime}}$ if $l\left(w w^{\prime}\right)=l(w)+l\left(w^{\prime}\right),\left(\underline{T}_{s}+1\right) \underline{T}_{s}=0$ if $s \in S ; M$ becomes a Q-vector space $M_{0}$ with basis $\left\{\underline{a}_{w} ; w \in \mathbf{I}_{*}\right\}$ and with $\mathfrak{H}_{0}$-module structure given by

$$
\begin{aligned}
& \underline{T}_{s} \underline{a}_{w}=\underline{a}_{s w} \text { if } s w=w s^{*}>w ; \\
& \underline{T}_{s} \underline{a}_{w}=\underline{a}_{s w s^{*}} \text { if } s w \neq w s^{*}>w ; \\
& \underline{T}_{s} \underline{a}_{w}=-a_{w} \text { if } s w<w .
\end{aligned}
$$

Here $s \in S, w \in \mathbf{I}_{*}$. We have the following result:

(a) There is a unique map $W \times \mathbf{I}_{*} \rightarrow \mathbf{I}_{*},(x, w) \mapsto x \circ$ w such that $\underline{T}_{x} \underline{a}_{w}=$ $\epsilon_{x, w} \underline{a}_{x \circ w}$ for any $x \in W, w \in \mathbf{I}_{*}$; here $\epsilon_{x, w}= \pm 1$ is a well-defined sign.

We argue by induction on $l(x)$. If $x=1$ we have $\underline{T}_{x} \underline{a}_{w}=\underline{a}_{w}$ so that $1 \circ w=$ $w, \epsilon_{1, w}=1$. Assume now that $x \neq 1$. Let $s \in S, x^{\prime} \in W$ be such that $x=s x^{\prime}>x^{\prime}$. By the induction hypothesis we have $\underline{T}_{x^{\prime}} \underline{a}_{w}= \pm \underline{a}_{u^{\prime}}$ for some $u^{\prime} \in \mathbf{I}_{*}$. Hence $\underline{T}_{x} \underline{a}_{w}= \pm \underline{T}_{s} \underline{a}_{u^{\prime}}$ and this equals $\pm \underline{a}_{u}$ for some $u \in \mathbf{I}_{*}$. This proves (a).

We show:

(b) $\underline{T}_{x} \underline{a}_{1}=(-1)^{l(x)} \epsilon(x \circ 1) \underline{a}_{x \circ 1}$ for any $x \in W$.

We argue by induction on $l(x)$. If $x=1$ we have $\underline{T}_{x} \underline{a}_{1}=\underline{a}_{1}$ hence $1 \circ 1=1$ and $l(x)=0, \epsilon(1 \circ 1)=\epsilon(1)=1$ and the result holds. Assume now that $x \neq 1$. Let $s \in S$, $x^{\prime} \in W$ be such that $x=s x^{\prime}>x^{\prime}$. By the induction hypothesis we have $\underline{T}_{x^{\prime}} \underline{a}_{1}=$ $(-1)^{l\left(x^{\prime}\right)} \epsilon(w) \underline{a}_{w}$ where $w=x^{\prime} \circ 1$. We have $\underline{T}_{x} \underline{a}_{1}=\underline{T}_{s} \underline{T}_{x^{\prime}} \underline{a}_{1}=(-1)^{l\left(x^{\prime}\right)} \epsilon(w) \underline{T}_{s} \underline{a}_{w}$. Now $\underline{T}_{s} \underline{a}_{w}=f(s, w) \underline{a}_{s \circ w}$ where $f(s, w)=1$ if $s w>w, f(s, w)=-1$ if $s w<w$. It is enough to prove that $(-1)^{l(x)} \epsilon(x \circ 1)=(-1)^{l\left(x^{\prime}\right)} \epsilon(w) f(s, w)$. Since $l(x)=l\left(x^{\prime}\right)+1$ it is enough to prove that $\epsilon(x \circ 1)=-\epsilon(w) f(s, w)$. We have $x \circ 1=s \circ w$ hence it is enough to prove that $\epsilon(s \circ w)=-\epsilon(w) f(s, w)$ or that $\epsilon(s w)=-\epsilon(w)$ if $s w=w s^{*}>w, \epsilon\left(s w s^{*}\right)=-\epsilon(w)$ if $s w \neq w s^{*}>w$. This is clear from the definition of $\epsilon$. This proves (b).

We define $\pi: W \rightarrow \mathbf{I}_{*}$ by $\pi(x)=x \circ 1$. We show:

(c) $\pi$ is surjective.

Let $w \in \mathbf{I}_{*}$. We show by induction on $l(w)$ that $w \in \pi(W)$. If $w=1$ we have $w=\pi(1)$. Assume now that $w \neq 1$. We can find $s \in S$ such that $s w<w$. Assume first that $s w=w s^{*}$. Then by the induction hypothesis we have $s w=x \circ 1$ for some $x \in W$ hence $\underline{T}_{s} \underline{T}_{x} \underline{a}_{1}= \pm \underline{T}_{s} \underline{a}_{s w}= \pm \underline{a}_{w}$; moreover, $\underline{T}_{s} \underline{T}_{x}$ equals $\underline{T}_{x}$ (if $s x>x$ ) or $\underline{T}_{s x}$ (if $\left.s x<x\right)$. Thus $w=x \circ 1$ or $w=s x \circ 1$.

Assume next that $s w \neq w s^{*}$. Then by the induction hypothesis we have $s w s^{*}=$ $x \circ 1$ for some $x \in W$ hence $\underline{T}_{s} \underline{T}_{x} \underline{a}_{1}= \pm \underline{T}_{s} \underline{a}_{s w s^{*}}= \pm \underline{a}_{w}$; moreover, $\underline{T}_{s} \underline{T}_{x}$ equals $\underline{T}_{x}$ (if $s x>x$ ) or $\underline{T}_{s x}$ (if $s x<x$ ). Thus $w=x \circ 1$ or $w=s x \circ 1$. This proves (c). 
1.9. For $x \in W$ we have $\underline{T}_{x} \underline{a}_{1}=\sum_{z \in \mathbf{I}_{*}} \underline{L}_{z}^{x} \underline{a}_{z}$ where $\underline{L}_{z}^{x}=\left.L_{z}^{x}\right|_{u=0} \in \mathbf{Z}$. Comparing with $1.8(\mathrm{~b})$ we see that $\underline{L}_{z}^{x}=(-1)^{l(x)} \epsilon(z)$ if $z=x \circ 1$ and $\underline{L}_{z}^{x}=0$ if $z \neq x \circ 1$. It follows that $\left.\tilde{L}_{z}^{x}\right|_{u^{-1}=0}=1$ if $z=x \circ 1$ and $\left.\tilde{L}_{z}^{x}\right|_{u^{-1}=0}=0$ if $z \neq x \circ 1$. Thus $0.2($ c) holds.

1.10. We show that the map $\mu: M \rightarrow \hat{\mathfrak{H}}$ is injective. It is enough to show that the elements $\left\{\mu\left(a_{z}\right) ; z \in \mathbf{I}_{*}\right\}$ are linearly independent. Assume that $\sum_{z \in \mathbf{I}_{*}} \xi_{z} \mu\left(a_{z}\right)=0$ where $\xi_{z} \in \mathbf{Q}(u)$ are zero for all but finitely many $z$ and $\xi_{z} \neq 0$ for some $z \in \mathbf{I}_{*}$. We can assume that $\xi_{z} \in \mathbf{Z}\left[u^{-1}\right]$ for all $z$ and $\left.\xi_{z}\right|_{u^{-1}=0} \neq 0$ for some $z=z_{0}$. We have $\sum_{z \in \mathbf{I}_{*}, x \in W} \xi_{z} \tilde{L}_{z}^{x} T_{x}=0$ hence $\sum_{z \in \mathbf{I}_{*}} \xi_{z} \tilde{L}_{z}^{x}=0$ for any $x \in W$. Setting $u^{-1}=0$ we deduce that $\left.\sum_{z \in \mathbf{I}_{*}} \xi_{z}\right|_{u^{-1}=0} n_{z}^{x}=0$ for any $x \in W$. By $0.2(\mathrm{c})$ this can be written as $\left.\xi_{\pi(x)}\right|_{u^{-1}=0}=0$ for any $x \in W$. By $1.8(\mathrm{c})$ we can find $x \in W$ such that $\pi(x)=z_{0}$. For this $x$ we have $\left.\xi_{z_{0}}\right|_{u^{-1}=0}=0$. This is a contradiction, Thus the injectivity of $\mu$ is proved. This completes the proof of Theorem 0.2 .

1.11. In the case where $W$ is of type $A_{1}$ with $S=\{s\}$ we have $\mu\left(a_{1}\right)=u^{-1} T_{s}+1$, $\mu\left(a_{s}\right)=(u-1) u^{-1} T_{s}$.

In the case where $W$ is of type $A_{2}$ with $S=\{s, t\}$ and $*=1$ we have:

$$
\begin{aligned}
& \mu\left(a_{1}\right)=u^{-3} T_{\text {sts }}+u^{-2} T_{s t}+u^{-2} T_{t s}+u^{-1} T_{s}+u^{-1} T_{t}+1, \\
& \mu\left(a_{s}\right)=(u-1)\left(u^{-3} T_{\text {sts }}+u^{-2} T_{s t}+u^{-1} T_{s}\right), \\
& \mu\left(a_{t}\right)=(u-1)\left(u^{-3} T_{\text {sts }}+u^{-2} T_{t s}+u^{-1} T_{t}\right), \\
& \mu\left(a_{\text {sts }}\right)=(u-1)\left(\left(u^{-1}+u^{-2}-u^{-3}\right) T_{\text {sts }}+u^{-1} T_{s t}+u^{-1} T_{t s}\right) .
\end{aligned}
$$

(See [L4, 32, 3.3].)

1.12. For $x \in W, z \in \mathbf{I}_{*}$ we set $\tilde{L}_{z}^{x}=(u-1)^{\phi(z)} \lambda_{z}^{x}$ where $\phi(z)$ is as in 1.5 and $\lambda_{z}^{x} \in \mathbf{Q}(u)$. We show:

(a) $\lambda_{z}^{x} \in \mathbf{Z}\left[u^{-1}\right]$ and $\overline{\lambda_{z}^{x}}=\left(-u^{2}\right)^{l(x)+(1 / 2)(l(z)-\phi(z))} \lambda_{z}^{x}$.

From the definitions we have $L_{z}^{1}=\delta_{1, z}$ hence $\tilde{L}_{z}^{1}=\delta_{1, z}$ and $\lambda_{z}^{1}=\delta_{1, z}$. From the formulas in 1.6 (with $s \in S$ ) we deduce (assuming $s x<x$ ):

$$
\begin{aligned}
& \lambda_{z}^{x}=\lambda_{s z}^{x}-u^{-1} \lambda_{z}^{s x} \text { if } s z=z s^{*}<z \\
& \lambda_{z}^{x}=u^{-1} \lambda_{z}^{s x}+\left(1-u^{-2}\right) \lambda_{s z}^{s x} \text { if } s z=z s^{*}>z \\
& \lambda_{z}^{x}=u^{-2} \lambda_{s z s^{*}}^{s x} \text { if } s z \neq z s^{*}>z \\
& \lambda_{z}^{x}=\lambda_{s z s^{*}}^{s x}+\left(1-u^{-2}\right) \lambda_{z}^{s x} \text { if } s z \neq z s^{*}<z .
\end{aligned}
$$

From this (a) follows by induction on $l(x)$.

1.13. In this subsection we give an application of the function $\epsilon: \mathbf{I}_{*} \rightarrow\{ \pm 1\}$ in 1.5. Let $E=\mathbf{Q}(u)$ viewed as an $\mathfrak{H}$-module in which $T_{x}(x \in W)$ acts as multiplication by $(-1)^{l(x)}($ sign representation of $\mathfrak{H})$. We define a $\mathbf{Q}(u)$-linear map $f: M \rightarrow E$ by $f\left(a_{z}\right)=\epsilon(z)$. We claim that $f$ is $\mathfrak{H}$-linear. It is enough to show that for any $w \in \mathbf{I}_{*}, s \in S$ we have:

$$
\begin{aligned}
& -\epsilon(w)=u \epsilon(w)+(u+1) \epsilon(s w) \text { if } s w=w s^{*}>w ; \\
& -\epsilon(w)=\left(u^{2}-u-1\right) \epsilon(w)+\left(u^{2}-u\right) \epsilon(s w) \text { if } s w=w s^{*}<w ; \\
& -\epsilon(w)=\epsilon\left(s w s^{*}\right) \text { if } s w \neq w s^{*}>w ; \\
& -\epsilon(w)=\left(u^{2}-1\right) \epsilon(w)+u^{2} \epsilon\left(s w s^{*}\right) \text { if } s w \neq w s^{*}<w .
\end{aligned}
$$

This follows from $1.5(\mathrm{a})$. 


\section{The Biregular Representation of $\mathfrak{H}$}

2.1. In this section we discuss the special case of Theorem 0.2 in which $W$ in 0.1 is replaced by $W^{2}=W \times W, S$ is replaced by $S^{2}=S \times\{1\} \cup\{1\} \times S$ and $*: W \rightarrow W$ is replaced by $*: W^{2} \rightarrow W^{2},(x, y) \mapsto(y, x)$. In this case we have $\mathbf{I}_{*}=\left\{(x, y) \in W^{2} ; x y=1\right\}$.

We use the notation $\mathfrak{H}, \hat{\mathfrak{H}}$ in reference to $W$. Let $\mathfrak{H}^{(2)}, \hat{\mathfrak{H}}^{(2)}$ be the objects analogous to $\mathfrak{H}, \hat{\mathfrak{H}}$ defined in terms of $W^{2}$ instead of $W$. Thus $\mathfrak{H}^{(2)}=\mathfrak{H} \otimes \mathfrak{H}$ with basis $\left\{T_{w} \otimes T_{w^{\prime}} ;\left(w, w^{\prime}\right) \in W^{2}\right\}$ which is the analogue for $\mathfrak{H}^{(2)}$ of the basis $\left\{T_{w} ; w \in W\right\}$ of $\mathfrak{H}$. We write $T_{w}$ (resp. $\left.T_{w}^{\prime}\right)$ instead of $T_{w} \otimes 1$ (resp. $\left.1 \otimes T_{w}\right)$. Then the basis element $T_{w} \otimes T_{w^{\prime}}$ is actually the product in $\mathfrak{H}^{(2)}$ of $T_{w}$ and $T_{w^{\prime}}^{\prime}$ in either order. In our case we have $M=\mathfrak{H}$ with the basis $\left\{a_{w, w^{-1}}=T_{w} ; w \in W\right\}$ viewed as an $\mathfrak{H}^{(2)}$-module in which the action of $T_{x} \otimes T_{y}$ is $T_{r} \mapsto T_{x} T_{r} T_{y^{-1}}$. (We refer to this as the biregular representation.) We can view $\mathfrak{H}^{(2)}$ as a subspace of $\hat{\mathfrak{H}}^{(2)}$ in an obvious way. The $\mathfrak{H}^{(2)}$-module structure on $\mathfrak{H}^{(2)}$ (left multiplication) extends in an obvious way to a $\mathfrak{H}^{(2)}$-module structure on $\hat{\mathfrak{H}}^{(2)}$. The following is a restatement of Theorem 0.2 in our case.

Corollary 2.2. (a) There exists a unique $\mathfrak{H}^{(2)}$-linear map $\mu: \mathfrak{H} \rightarrow \hat{\mathfrak{H}}^{(2)}$ (where $\mathfrak{H}, \hat{\mathfrak{H}}^{(2)}$ are viewed as $\mathfrak{H}^{(2)}$-modules as above) such that $\mu(1)=\sum_{w \in W} u^{-2 l(w)} T_{w} \otimes$ $T_{w} \in \hat{\mathfrak{H}}^{(2)}$. Moreover, $\mu$ is an isomorphism of $\mathfrak{H}$ onto the $\mathfrak{H}^{(2)}$-submodule of $\hat{\mathfrak{H}}^{(2)}$ generated by $\mu(1)$.

(b) Let $z \in W$. We set $\mu\left(T_{z}\right)=\sum_{(x, y) \in W^{2}} N_{z}^{x, y} T_{x} \otimes T_{y}$ where $N_{z}^{x, y} \in \mathbf{Q}(u)$. For any $(x, y) \in W^{2}$ we have $N_{z}^{x, y} \in \mathbf{Z}\left[u^{-1}\right]$, hence we can define $n_{z}^{x, y}=\left.N_{z}^{x, y}\right|_{u^{-1}=0} \in$ $\mathbf{Z}$.

(c) There is a unique surjective function $\pi: W^{2} \rightarrow W$ such that for $(x, y) \in W$, $z \in W$ we have $n_{z}^{x, y}=1$ if $z=\pi(x, y), n_{z}^{x, y}=0$ if $z \neq \pi(x, y)$. (Note that $\pi(1,1)=1$.)

In the remainder of this section we shall indicate a proof of a part of the corollary which is somewhat different from that of Theorem 0.2 .

2.3. Let $\tau: \mathfrak{H} \rightarrow \mathbf{Q}(u)$ be the $\mathbf{Q}(u)$-linear map such that $\tau\left(T_{x}\right)=0$ if $x \neq 1$, $\tau\left(T_{1}\right)=1$. For $x, y \in W$ we have $\tau\left(T_{x} T_{y}\right)=0$ if $x y \neq 1, \tau\left(T_{x} T_{y}\right)=u^{2 l(x)}$ if $x y=1$. (See [L3, 10.4(a)].) It follows that for $x, y$ in $W$ we have

$$
T_{x} T_{y}=\sum_{z \in W} \tau\left(T_{x} T_{y} T_{z}\right) u^{-2 l(z)} T_{z^{-1}} .
$$

Since $T_{x} \mapsto T_{x^{-1}}$ defines an antiautomorphism of $\mathfrak{H}$ we have

$$
T_{y^{-1}, x^{-1}}=\sum_{z \in W} \tau\left(T_{x} T_{y} T_{z}\right) u^{-2 l(z)} T_{z}=\sum_{z \in W} \tau\left(T_{y^{-1}} T_{x^{-1}} T_{z^{-1}}\right) u^{-2 l(z)} T_{z}
$$

hence

$$
\tau\left(T_{x} T_{y} T_{z}\right)=\tau\left(T_{y^{-1}} T_{x^{-1}} T_{z^{-1}}\right)
$$

for any $x, y, z$ in $W$. By [L3, 10.4(b)] we have $\tau\left(h h^{\prime}\right)=\tau\left(h^{\prime} h\right)$ for any $h, h^{\prime}$ in $\mathfrak{H}$. In particular for $x, y, z$ in $W$ we have

$$
\tau\left(T_{x} T_{y} T_{z}\right)=\tau\left(T_{y} T_{z} T_{x}\right)=\tau\left(T_{z} T_{x} T_{y}\right)
$$


Lemma 2.4. (a) For $x, y, z$ in $W$ we set $p_{x, y, z}=\tau\left(T_{x} T_{y} T_{z}\right) u^{-2 l(z)-2 l(y)}, p_{x, y, z}^{\prime}=$ $\tau\left(T_{x} T_{y} T_{z}\right) u^{-2 l(x}$. We have $p_{x, y, z} \in d_{x, y, z}+u^{-1} \mathbf{Z}\left[u^{-1}\right], p_{x, y, z}^{\prime} \in d_{x, y, z}^{\prime}+u \mathbf{Z}[u]$ where $d_{x, y, z} \in\{0,1\}, d_{x, y, z}^{\prime} \in\{0, \pm 1\}$. Moreover, $p_{x, y, z}^{\prime}=(-1)^{l(x)+l(y)+l(z)} \overline{p_{x, y, z}}$ hence $d_{x, y, z}^{\prime}=(-1)^{l(x)+l(y)+l(z)} d_{x, y, z}$.

(b) Let $y, z$ be in $W$. There is exactly one $x \in W$ (denoted by $y * z$ ) such that $d_{x, y, z}=1$ (or equivalently such that $d_{x, y, z}^{\prime}= \pm 1$ ). For all other $x$ we have $d_{x, y, z}=d_{x, y, z}^{\prime}=0$.

We argue by induction on $l(z)$. If $z=1$ we have $p_{x, y, z}=\tau\left(T_{x} T_{y}\right) u^{-2 l(y)}=\delta_{x, y}$, $p_{x, y, z}^{\prime}=\tau\left(T_{x} T_{y}\right) u^{-2 l(x)}=\delta_{x, y}$. Hence (a),(b) hold with $y * z=y^{-1}$. (We have $\left.\delta_{x, y}=(-1)^{l(x)+l(y)} \delta_{x, y} \cdot\right)$

Assume now that $l(z) \geq 1$. We write $z=s z^{\prime}, s \in S, l\left(z^{\prime}\right)=l(z)-1$. If $y s>y$ we have by the induction hypothesis

$$
\begin{gathered}
p_{x, y, z}=\tau\left(T_{x} T_{y} T_{s} T_{z^{\prime}}\right) u^{-2 l\left(z^{\prime}\right)-2 l(y)-2}=p_{x, y s, z^{\prime}} \in d_{x, y s, z^{\prime}}+u^{-1} \mathbf{Z}\left[u^{-1}\right], \\
p_{x, y, z}^{\prime}=\tau\left(T_{x} T_{y} T_{s} T_{z^{\prime}}\right) u^{-2 l(x)}=p_{x, y s, z^{\prime}}^{\prime} \in d_{x, y s, z^{\prime}}^{\prime}+u \mathbf{Z}[u]
\end{gathered}
$$

hence the result holds: we have $d_{x, y, z}=d_{x, y s, z^{\prime}}, d^{\prime} x, y, z=d_{x, y s, z^{\prime}}^{\prime}, y * z=(y s) *(s z)$. (We use that $(-1)^{l(x)+l(y)+l(z)}=(-1)^{l(x)+l(y s)+l\left(z^{\prime}\right)}$.)

If $y s<y$ we have by the induction hypothesis

$$
\begin{aligned}
& p_{x, y, z}=\tau\left(T_{x} T_{y} T_{s} T_{z^{\prime}}\right) u^{-2 l\left(z^{\prime}\right)-2 l(y)-2} \\
& =p_{x, y s, z^{\prime}} u^{-2}+p_{x, y, z^{\prime}} u^{-2}\left(u^{2}-1\right) \in d_{x, y, z^{\prime}}+u^{-1} \mathbf{Z}\left[u^{-1}\right], \\
& p_{x, y, z}^{\prime}=\tau\left(T_{x} T_{y} T_{s} T_{z^{\prime}}\right) u^{-2 l(x)} \\
& =p_{x, y s, z^{\prime}}^{\prime} u^{2}+p_{x, y, z^{\prime}}^{\prime}\left(u^{2}-1\right) \in-d_{x, y, z^{\prime}}^{\prime}+u \mathbf{Z}[u]
\end{aligned}
$$

hence the result holds: we have $d_{x, y, z}=d_{x, y, z^{\prime}}, d_{x, y, z}^{\prime}=-d_{x, y, z^{\prime}}^{\prime}, y * z=y *(s z)$.

(We use that

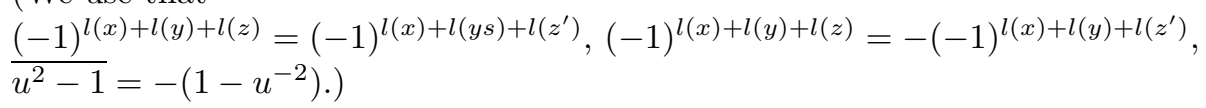

2.5. For $a \in W$ we show that

$$
T_{a} X=T_{a^{-1}}^{\prime} X
$$

We have

$$
\begin{aligned}
T_{a} X & =\sum_{w, z \in W} u^{-2 l(w)-2 l(z)} \tau\left(T_{a} T_{w} T_{z}^{-1}\right) T_{z} T_{w}^{\prime} \in \hat{\mathfrak{H}}^{(2)}, \\
T_{a^{-1}}^{\prime} X & =\sum_{w, z \in W} u^{-2 l(w)-2 l(z)} \tau\left(T_{a^{-1}} T_{w} T_{z^{-1}}\right) T_{w} T_{z}^{\prime} \in \hat{\mathfrak{H}}^{(2)} .
\end{aligned}
$$

Making the change of variable $(w, z) \mapsto(z, w)$ in the last sum we obtain

$$
T_{a^{-1}}^{\prime} X=\sum_{w, z \in W} u^{-2 l(w)-2 l(z)} \tau\left(T_{a^{-1}} T_{z} T_{w^{-1}}\right) T_{z} T_{w}^{\prime}
$$

It remains to show that

$$
\tau\left(T_{a^{-1}} T_{z} T_{w^{-1}}\right)=\tau\left(T_{a} T_{w} T_{z^{-1}}\right) .
$$

Indeed, by $2.3(\mathrm{a})$ the left-hand side is equal to $\tau\left(T_{z^{-1}} T_{a} T_{w}\right)$ and by $2.3(\mathrm{~b})$ this is equal to the right-hand side. 
2.6. We give an alternative proof of the existence of $\mu$ in Corollary 2.2. For any $a \in W$ we set $X_{a}=T_{a} X=T_{a^{-1}}^{\prime} X \in \hat{\mathfrak{H}}^{(2)}$; see $2.5(\mathrm{a})$. Thus, $X_{1}=X$. We define a $\mathbf{Q}(u)$-linear map $\mu: \mathfrak{H} \rightarrow \hat{\mathfrak{H}}^{(2)}$ by $T_{a} \mapsto X_{a}$ for all $a \in W$. For $h \in \mathfrak{H}, r \in W$ we have $\mu\left(T_{r} h\right)=T_{r} \mu(h)$ (using the description $\left.X_{a}=T_{a^{-1}}^{\prime} X\right)$ and $\mu\left(h T_{r^{-1}}\right)=T_{r}^{\prime} \mu(h)$ (using the description $\left.X_{a}=T_{a} X\right)$. It follows that $\mu$ is $\mathfrak{H}^{(2)}$-linear.

In our case $\pi: W^{2} \rightarrow W$ is given by $\pi(x, y)=\left(y *\left(x^{-1}\right),\left(y *\left(x^{-1}\right)\right)^{-1}\right.$.

2.7. In the case where $W$ is of type $A_{1}$ with $S=\{s\}$ we have:

$$
\begin{aligned}
& \mu\left(T_{1}\right)=T_{1} \otimes T_{1}+u^{-2} T_{s} \otimes T_{s}, \\
& \mu\left(T_{s}\right)=T_{1} \otimes T_{s}+T_{s} \otimes T_{1}+\left(1-u^{-2}\right) T_{s} \otimes T_{s} .
\end{aligned}
$$

\section{3. $\Gamma$-Equivariant VECTOR BUNDLES ON $\Gamma$}

3.1. Let $\Gamma$ be a finite group. Let $K_{\Gamma}(\Gamma)$ be the Grothendieck group of $\Gamma$-equivariant (complex) vector bundles on $\Gamma$ where $\Gamma$ acts on $\Gamma$ by conjugation. For $x \in \Gamma$ let $\Gamma_{x}=Z_{\Gamma}(x)$ and let $\operatorname{Irr} \Gamma_{x}$ be a set of representatives for the isomorphism classes of irreducible representations of $\Gamma_{x}$ over $\mathbf{C}$. For any $x \in \Gamma$ and any $\rho \in \operatorname{Irr} \Gamma_{x}$ there is a unique (up to isomorphism) $\Gamma$-equivariant vector bundle $E_{x, \rho}$ on $\Gamma$ such that the support of $E_{x, \rho}$ is the conjugacy class of $x$ and is such that the action of $\Gamma_{x}$ on the fibre of $E_{x, \rho}$ is isomorphic to $\rho$. Let $\underline{\Gamma}$ be a set of representatives for the conjugacy classes in $\Gamma$. Let $\mathfrak{M}(\Gamma)=\left\{(x, \rho) ; x \in \underline{\Gamma}, \rho \in \operatorname{Irr} \Gamma_{x}\right\}$. The classes of $E_{x, \rho}$ (with $(x, \rho) \in \mathfrak{M}(\Gamma)$ ) form a Z-basis of $K_{\Gamma}(\Gamma)$.

Following Kottwitz [Ko] we consider the element $\kappa \in K_{\Gamma}(\Gamma)$ defined by

$$
\kappa=\sum_{(x, \rho) \in \mathfrak{M}(\Gamma)} \sum_{s \in \Gamma ; s^{2}=x} \frac{\left|Z_{\Gamma_{x}}\right|}{\left|\Gamma_{x}\right|}\left(1:\left.\rho\right|_{Z_{\Gamma_{x}}(s)}\right) E_{x, \rho}
$$

where $\left(1:\left.\rho\right|_{Z_{\Gamma_{x}}(s)}\right)$ denotes the multiplicity of the unit representation of $Z_{\Gamma_{x}}(s)$.

Proposition 3.2. Define $V=\alpha_{!} \mathbf{C}$ where $\alpha: \Gamma \rightarrow \Gamma$ is $g \mapsto g^{2}$. Note that $V$ is a $\Gamma$-equivariant vector bundle on $\Gamma$. We have $V=\kappa$ in $K_{\Gamma}(\Gamma)$.

Let $\Gamma^{(2)}=\{(g, h) \in \Gamma \times \Gamma ; g h=h g\}$. For any $\Gamma$-equivariant vector bundle $\mathcal{V}$ on $\Gamma$ we define $\phi_{\mathcal{V}}: \Gamma^{(2)} \rightarrow \mathbf{C}$ as follows: $\phi_{\mathcal{V}}(g, h)$ is trace of the action of $h$ on the fibre of $\mathcal{V}$ at $g$. For example, if $(x, \rho) \in \mathfrak{M}(\Gamma)$, we have

$$
\phi_{E_{x, \rho}}(g, h)=\left|\Gamma_{x}\right|^{-1} \sum_{a \in \Gamma ; a g a^{-1}=x} \operatorname{tr}\left(a h a^{-1}, \rho\right) .
$$

Note that $\mathcal{V} \rightarrow \phi_{\mathcal{V}}$ induces an injective linear map from the vector space $\mathbf{C} \otimes K_{\Gamma}(\Gamma)$ into the vector spaces of functions $\Gamma^{(2)} \rightarrow \mathbf{C}$; see [L2. Hence it suffices to show that $\phi_{V}=\phi_{\kappa}$. For $(g, h) \in \Gamma^{(2)}$ we have

$$
\begin{aligned}
& \phi_{\kappa}(g, h)=\sum_{x \in \Gamma, \rho \in \operatorname{Irr} \Gamma_{x}} \frac{\left|\Gamma_{x}\right|}{|\Gamma|} \sum_{s \in \Gamma ; s^{2}=\xi} \frac{\left|Z_{\Gamma_{x}}(s)\right|}{\left|\Gamma_{x}\right|}\left(1:\left.\rho\right|_{\Gamma_{x} \cap \Gamma_{s}}\right) \phi_{E_{x, \rho}}(g, h) \\
& =\sum_{x \in \Gamma, \rho \in \operatorname{Irr} \Gamma_{x}}|\Gamma|^{-1} \sum_{s \in \Gamma ; s^{2}=x} \sum_{u \in \Gamma_{x} \cap \Gamma_{s}} \operatorname{tr}\left(u^{-1}, \rho\right)\left|\Gamma_{x}\right|^{-1} \sum_{a \in \Gamma ; a g a^{-1}=x} \operatorname{tr}\left(a h a^{-1}, \rho\right) \\
& =\sum_{x \in \Gamma}|\Gamma|^{-1} \sum_{s \in \Gamma ; s^{2}=x} \sum_{u \in \Gamma_{x} \cap \Gamma_{s}}\left|\Gamma_{x}\right|^{-1} \sum_{a \in \Gamma ; a g a^{-1}=x}\left|\left\{z \in G_{x} ; z a h a^{-1} z^{-1}=u^{-1}\right\}\right| .
\end{aligned}
$$


Setting $s^{\prime}=a^{-1} s a, u^{\prime}=a^{-1} u a, z^{\prime}=a^{-1} z a$ we obtain

$$
\begin{aligned}
& \phi_{\kappa}(g, h)=\sum_{s^{\prime} \in \Gamma ; s^{\prime 2}=g} \sum_{u^{\prime} \in \Gamma_{g} \cap \Gamma_{s^{\prime}}}\left|\Gamma_{g}\right|^{-1}\left|\left\{z^{\prime} \in G_{g} ; z^{\prime} h z^{\prime-1}=u^{\prime-1}\right\}\right| \\
& =\sum_{s^{\prime} \in \Gamma ; s^{\prime 2}=g}\left|\Gamma_{g}\right|^{-1}\left|\left\{z^{\prime} \in G_{g} ; z^{\prime} h z^{\prime-1} \in G_{g} \cap G_{s^{\prime}}\right\}\right| .
\end{aligned}
$$

Setting $\tilde{s}=z^{\prime-1} s^{\prime} z^{\prime}$ we obtain

$$
\phi_{\kappa}(g, h)=\left|\left\{\tilde{s} \in \Gamma ; \tilde{s}^{2}=g, \tilde{s} h=h \tilde{s}\right\}\right| .
$$

From the definitions we have

$$
\phi_{V}(g, h)=\left|\left\{\tilde{s} \in \Gamma ; \tilde{s}^{2}=g, \tilde{s} h=h \tilde{s}\right\}\right| .
$$

The proposition is proved.

3.3. As in [L2, 2.5], any $(y, \sigma) \in \mathfrak{M}(\Gamma)$ defines a C-linear function $\chi_{y, \sigma}: \mathbf{C} \otimes K_{\Gamma}(\Gamma) \rightarrow \mathbf{C}$ by the rule

$$
\chi_{y, \sigma}(U)=(\operatorname{dim} \sigma)^{-1} \sum_{\gamma \in G_{y}} \operatorname{tr}\left(y, U_{\gamma}\right) \operatorname{tr}(\gamma, \sigma)
$$

for any $\Gamma$-equivariant vector bundle. (This is in fact an algebra homomorphism for the algebra structure defined in [L2, 2.2].) Moreover, if $(x, \rho) \in M(\Gamma)$, then

$$
\chi_{y, \sigma}\left(E_{x, \rho}\right)=\frac{\left|\Gamma_{y}\right|}{\operatorname{dim} \sigma}\left\{(x, \rho),\left(y, \sigma^{*}\right)\right\}
$$

where $\{$,$\} is the nonabelian Fourier transform matrix of [L1] and \sigma^{*} \in \operatorname{Irr} \Gamma_{y}$ is isomorphic to the dual of $\sigma$. We compute $\chi_{y, \sigma}(V)$ where $V$ is as in 3.2 and $\sigma$ has Frobenius-Schur indicator 1. By the proof of 3.2 we have

$$
\begin{aligned}
& \chi_{y, \sigma}(V)=(\operatorname{dim} \sigma)^{-1} \sum_{\gamma \in \Gamma_{y}} \operatorname{tr}\left(y, V_{\gamma}\right) \operatorname{tr}(\gamma, \sigma) \\
& =(\operatorname{dim} \sigma)^{-1} \sum_{\gamma \in \Gamma_{y}, \tilde{s} \in \Gamma_{y} ; \tilde{s}^{2}=\gamma} \operatorname{tr}(\gamma, \sigma)=(\operatorname{dim} \sigma)^{-1} \sum_{\tilde{s} \in \Gamma_{y}} \operatorname{tr}\left(\tilde{s}^{2}, \sigma\right)=\frac{\left|\Gamma_{y}\right|}{\operatorname{dim} \sigma} .
\end{aligned}
$$

Combining this with (a) we see that

$$
\sum_{(x, \rho)}\{(x, \rho),(y, \sigma)\} \text { mult. of } E_{x, \rho} \text { in } V=1 .
$$

\section{Some Applications of Theorem 0.2}

4.1. Let $A$ be a finite dimensional split semisimple algebra over a field $K$. Let $\operatorname{Mod} A$ be the category of $A$-modules of finite dimension over $K$. For $E^{\prime} \in \operatorname{Mod} A$ let $A^{E^{\prime}}$ be the sum of the simple two-sided ideals $I$ of $A$ such that $I E^{\prime} \neq 0$. For $E, E^{\prime}$ in $\operatorname{Mod} A$ let $E_{E^{\prime}}=A^{E^{\prime}} E=\sum_{f \in \operatorname{Hom}_{A}\left(E^{\prime}, E\right)} f\left(E^{\prime}\right)$. We have the following result.

(a) Let $E \in \operatorname{Mod} A$ and let $\mathcal{X} \in A$. We have a canonical $K$-linear isomorphism $\alpha: \mathcal{X} E \stackrel{\sim}{\rightarrow} \operatorname{Hom}_{A}(A \mathcal{X}, E)$. Moreover, $\mathcal{X} E \subset E_{A \mathcal{X}}$. We have $A \mathcal{X} A \subset A^{A \mathcal{X}}$.

(Note that $A \mathcal{X}$ is a left ideal of $A$ hence an object of $\operatorname{Mod} A$.) For $e \in \mathcal{X} E$ we define $f_{e}: A \mathcal{X} \rightarrow E$ by $f_{e}(a \mathcal{X})=a e, a \in A ; f_{e}$ is well defined: if $a, a^{\prime} \in A$ satisfy 
$a \mathcal{X}=a^{\prime} \mathcal{X}$, then $a e-a^{\prime} e=a \mathcal{X} e_{0}-a^{\prime} \mathcal{X} e_{0}=0$ where $e=\mathcal{X} e_{0}, e_{0} \in E$. Now $e \mapsto f_{e}$ is a $K$-linear map $\alpha: \mathcal{X} E \rightarrow \operatorname{Hom}_{A}(A \mathcal{X}, E)$ which is clearly injective. We have $\operatorname{dim}_{K}(\mathcal{X} E)=\operatorname{dim}_{K} \operatorname{Hom}_{A}(A \mathcal{X}, E)$. (We can assume that $A$ is a simple $K$-algebra and $E$ is a simple $A$-module. Thus we can assume that for some $K$-vector space $V$ of finite dimension we have $A=\operatorname{End}(V)$ and $E=V$ is viewed as an $A$-module in an obvious way. In this case the desired statement is easily verified.) It follows that $\alpha$ is an isomorphism.

We prove the second statement of (a). For $e \in \mathcal{X} E$ we have $f_{e}(\mathcal{X})=e$. Since $f_{e} \in \operatorname{Hom}_{A}(A \mathcal{X}, E)$ we see that $e \in E_{A \mathcal{X}}$, proving the second statement of (a). Applying this to $E=A$ viewed as an object of $\operatorname{Mod} A$ under left multiplication we see that $\mathcal{X} A \subset A_{A \mathcal{X}}$. We now observe that $A_{A \mathcal{X}} \subset A^{A \mathcal{X}}$. Hence $\mathcal{X} A \subset A^{A \mathcal{X}}$ and $A \mathcal{X} A \subset A A^{A \mathcal{X}}=A^{A \mathcal{X}}$. This proves the third statement of (a).

In the remainder of this section we assume that $W$ is a Weyl group and $*=1$.

Theorem 4.2. Let $M \in \operatorname{Mod} \mathfrak{H}, X \in \mathfrak{H}$ be as in 0.1. Let $E \in \operatorname{Mod} \mathfrak{H}$. We have canonically $X E \cong \operatorname{Hom}_{\mathfrak{H}}(M, E)$. Moreover, $X E \subset E_{M}$ and $\mathfrak{H} X \mathfrak{H} \subset \mathfrak{H}^{M}$ (notation of 4.1).

We apply 4.1 with $K=\mathbf{Q}(u), A=\mathfrak{H}=\hat{\mathfrak{H}}, \mathcal{X}=X$ and we use Theorem 0.2. The theorem follows.

If $E$ is a simple object of $\operatorname{Mod} \mathfrak{H}$ then $\operatorname{dim}_{K} \operatorname{Hom}_{\mathfrak{H}}(M, E)$ is known from the work of Kottwitz [Ko; indeed, by [LV], the specialization of our $M$ at $u=1$ is (noncanonically) isomorphic to a $W$-module explicitly computed in $\mathrm{Ko}$. In particular, using the theorem we see that (a),(b) below hold.

(a) If $W$ is of type $A_{n}$ and $E$ is a simple $\mathfrak{H}$-module then $\operatorname{dim}_{K}(X E)=1$; in particular, $E$ contains a canonical line.

(b) If $W$ is of type $B_{n}$ or $D_{n}$ and $E$ is a simple $\mathfrak{H}$-module then $\operatorname{dim}_{K}(X E)$ is a power of 2 if $E$ is a special representation (see [L1]) and $X E=0$ if $E$ is a nonspecial representation.

4.3. Let $\mathcal{A}=\mathbf{Z}\left[u, u^{-1}\right] \subset K$. Let $\mathcal{H}$ be the $\mathcal{A}$-subalgebra of $\mathfrak{H}$ with basis $\left\{T_{w} ; w \in\right.$ $W\} ; \mathcal{H}$ is the same as the $\mathcal{A}$-algebra defined in [L3, 3.2] except that $T_{w}, v$ of [L3, 3.2] are the same as $u^{-l(w)} T_{w}, u$ of this paper. (When we refer to [L3] we assume that $L=l$ as in [L3, 15.1].)

Let $J$ be the asymptotic Hecke algebra (over $\mathbf{Z}$ ) with basis $\left\{t_{z} ; z \in W\right\}$ associated to $W$; see [L3, §18]. Let $\mathbf{J}=\mathbf{Q} \otimes J,{ }_{K} J=K \otimes \mathbf{J}$; these are split semisimple algebras.

Let $\left\{c_{w} ; w \in W\right\}$ be the $\mathcal{A}$-basis of $\mathcal{H}$ as in [L3, 5.2]. For $x, y, z$ in $W$ let $h_{x, y, z} \in \mathcal{A}$ be as in [L3, 13.1]. For $x, y$ in $W$ we write $x \sim y$ if $x, y$ are in the same left cell. For $x \in W$ let $a(x) \in \mathbf{N}$ be as in [L3, 13.6]. Let $\mathcal{D} \subset W$ be as in [L3, 14.1]. The $K$-linear map $\psi: \mathfrak{H} \rightarrow{ }_{K} \mathbf{J}$ given by $c_{x} \mapsto \sum_{d \in \mathcal{D}, z \in W ; d \sim z^{-1}} h_{x, d, z} t_{z}$ is a $K$ algebra isomorphism (see [L3, 18.8]).

For any $\mathcal{E} \in \operatorname{Mod} \mathbf{J}$ we set ${ }_{K} \mathcal{E}=K \otimes \mathcal{E} \in \operatorname{Mod}\left({ }_{K} \mathbf{J}\right)$; let $\mathcal{E}_{u}$ be the $\mathfrak{H}$-module corresponding to ${ }_{K} \mathcal{E}$ under $\phi$. Let $\mathcal{M} \in \operatorname{Mod}(\mathbf{J})$ be such that $\mathcal{M}_{u} \cong M$.

From 4.2 we deduce the following result.

Corollary 4.4. Let $\mathcal{E} \in \operatorname{Mod} \mathbf{J}$. We have

$$
\operatorname{dim}_{K}\left(\psi(X)\left({ }_{K} \mathcal{E}\right)\right)=\operatorname{dim}_{K} \operatorname{Hom}_{\mathfrak{H}}\left(M, \mathcal{E}_{u}\right) .
$$

Moreover, $\psi(X)\left({ }_{K} \mathcal{E}\right) \subset\left({ }_{K} \mathcal{E}\right)_{K} \mathcal{M}$. 
4.5. For $x, y, z$ in $W$ we have

(a) $h_{x, y, z}=\gamma_{x, y, z^{-1}} u^{a(z)}+$ lower powers of $u$ where $\gamma_{x, y, z^{-1}} \in \mathbf{N}$;

see [L3, 13.6]. For $x \in W$ we have $u^{-l(w)} T_{w}=\sum_{y \in W} s_{y, w} c_{y}$ where

(b) $s_{y, w} \in u^{-1} \mathbf{Z}\left[u^{-1}\right]$ for all $y \neq w$ and $s_{w, w}=1$.

Proposition 4.6. Let $Z$ be a left cell of $W$ and let $a=a(h)$ for any $h \in Z$. Let $\xi \in W$ be such that $\xi^{-1} \in Z$. We have

$$
\psi(X) t_{\xi}=\sum_{z \in Z} r_{z} t_{z} t_{\xi}
$$

where $r_{z}=u^{a}+\sum_{i<a} n_{i, z} u^{i}$ and $n_{i, z} \in \mathbf{Z}$ are zero for all but finitely many $i$.

From the definitions we have

$$
\begin{aligned}
& \psi(X) t_{\xi}=\sum_{w \in W} \psi\left(u^{-l(w)} T_{w}\right) t_{\xi}=\sum_{y, w \in W} s_{y, w} \psi\left(c_{y}\right) t_{\xi} \\
& =\sum_{y, w, z \in W, d \in \mathcal{D} ; a(d)=a(z)} s_{y, w} h_{y, d, z} t_{z} t_{\xi} .
\end{aligned}
$$

By [L3, 14.2], in the last sum we can assume that $z \in Z$ and that $d \in Z$. Hence

$$
\psi(X) t_{\xi}=\sum_{y, w \in W, z \in Z, d \in \mathcal{D} \cap Z} s_{y, w} h_{y, d, z} t_{z} t_{\xi} .
$$

Using 4.5(a),(b), we see that

$$
\psi(X) t_{\xi}=\sum_{y \in W, z \in Z, d \in \mathcal{D} \cap Z} \gamma_{y, d, z^{-1}} u^{a} t_{z} t_{\xi}+\text { lower powers of } u .
$$

Using [L3, 14.2], we see that $\gamma_{y, d, z^{-1}}$ is 1 if $y=z$ and is 0 otherwise. Thus we have

$$
\psi(X) t_{\xi}=\sum_{z \in Z} u^{a} t_{z} t_{\xi}+\text { lower powers of } u .
$$

The proposition is proved.

Corollary 4.7. Let $Z, Z^{\prime}$ be two left cells of $W$ such that $Z \cap Z^{\prime-1} \neq \emptyset$. We have $\sum_{z \in Z \cap Z^{\prime-1}} t_{z} \in \mathbf{J}^{\mathcal{M}}$.

Let $a=a(w)$ for any $w \in Z$. Let $d$ (resp. $d^{\prime}$ ) be the unique element of $\mathcal{D} \cap Z$ (resp. $\left.\mathcal{D} \cap Z^{\prime}\right)$. From 4.2 we deduce (using $\psi$ ) that $\psi(X) t_{d} \in\left({ }_{K} \mathbf{J}\right)^{K} \mathcal{M}=K \otimes\left(\mathbf{J}^{\mathcal{M}}\right)$. Using now 4.6 we deduce that $\sum_{z \in Z}\left(u^{a}+\sum_{i<a} n_{i, z} u^{i}\right) t_{z} \in K \otimes\left(\mathbf{J}^{\mathcal{M}}\right)$. It follows that $\sum_{z \in Z} t_{z} \in \mathbf{J}^{\mathcal{M}}$ hence $t_{d^{\prime}} \sum_{z \in Z} t_{z} \in \mathbf{J}^{\mathcal{M}}$. We now note that $t_{d^{\prime}} \sum_{z \in Z} t_{z}=$ $\sum_{z \in Z \cap Z^{\prime-1}} t_{z}$. The corollary is proved.

4.8. We now assume in addition that $W$ is of type $A_{n}, B_{n}$ or $D_{n}$. Then, by $4.2(\mathrm{a}),(\mathrm{b})$, the two-sided ideal $\mathbf{J}^{\mathcal{M}}$ of $\mathbf{J}$ is the sum of the simple two-sided ideals corresponding to the various special representations of $W$. The dimension of this sum is equal to the number of pairs of left cells $Z, Z^{\prime}$ such that $Z \cap Z^{\prime-1} \neq \emptyset$. Hence in this case, from 4.7 we deduce:

(a) the elements $\sum_{z \in Z \cap Z^{\prime-1}} t_{z}$ for various $Z, Z^{\prime}$ as above form a $\mathbf{Q}$-basis of $\mathbf{J}^{\mathcal{M}}$. It follows that for any two-sided cell $c$ of $W$ and any left cell $Z$ contained in $c$;

(b) the elements $\sum_{z \in Z \cap Z^{\prime-1}} t_{z}$ (for various left cells $Z^{\prime}$ contained in c) form a $\mathbf{Q}$-basis of the unique left $\mathbf{J}$-submodule of $\bigoplus_{z \in Z} t_{z}$ isomorphic to the special representation of $\mathbf{J}$ associated to $c$. 
4.9. For irreducible $W$ of exceptional type, the elements described in 4.8(a) do not span the $\mathbf{Q}$-vector space $\mathbf{J}^{\mathcal{M}}$. For example, if $W$ is of type $G_{2}$, that is, a dihedral group with generators $s_{1}, s_{2}$ such that $\left(s_{1} s_{2}\right)^{6}=1$, then (a) provides only 6 elements while $\operatorname{dim} \mathbf{J}^{\mathcal{M}}=8$. If we write $t_{12 \ldots}$ instead of $t_{s_{1} s_{2} \ldots}, t_{21 \ldots}$ instead of $t_{s_{2} s_{1} \ldots}$ and $t_{\emptyset}$ instead of $t_{\text {unit element }}$, then the following 8 elements form a $\mathbf{Q}$-basis of $\mathbf{J}^{\mathcal{M}}$ :

$$
t_{\emptyset}, t_{1}+t_{12121}, t_{121}, t_{2}+t_{21212}, t_{212}, t_{12}+t_{1212}, t_{21}+t_{2121}, t_{121212} .
$$

This, together with 4.8(a), suggests that for any $W, \mathbf{J}^{\mathcal{M}}$ admits a $\mathbf{Q}$-basis consisting of $\mathbf{N}$-linear combinations of elements $t_{z}$.

4.10. Let $M_{\mathcal{A}}$ be the $\mathcal{A}$-submodule of $M$ with basis $\left\{a_{w} ; w \in \mathbf{I}_{*}\right\}$. Note that the $\mathfrak{H}$ module structure on $M$ restricts to an $\mathcal{H}$-module structure on $M_{\mathcal{A}}$. For any $\lambda \in \mathbf{C}^{*}$ we regard $\mathbf{C}$ as an $\mathcal{A}$-module via $u \mapsto \lambda$. We can then form $M_{\lambda}=\mathbf{C} \otimes_{\mathcal{A}} M_{\mathcal{A}}, \mathcal{H}_{\lambda}=$ $\mathbf{C} \otimes_{\mathcal{A}} \mathcal{H}$ and $M_{\lambda}$ becomes a module over the $\mathbf{C}$-algebra $\mathcal{H}_{\lambda}$. Let $X_{\lambda}=1 \otimes X \in \mathcal{H}_{\lambda}$ where $X$ is as in 0.1. Now the assignment $a_{z} \mapsto \sum_{x \in W} \tilde{L}_{z}^{x} T_{z}$ in 1.7 defines an $\mathcal{H}$-linear map $\mu_{\mathcal{A}}: M_{\mathcal{A}} \rightarrow \mathcal{H}$ such that $\mu_{\mathcal{A}}\left(a_{1}\right)=X$; by extension of scalars this gives rise to an $\mathcal{H}_{\lambda}$-linear map $\mu_{\lambda}: M_{\lambda} \rightarrow \mathcal{H}_{\lambda}$ such that $\mu_{\lambda}\left(a_{1}\right)=X_{\lambda}$. Now, if $\lambda \neq-1$, the $\mathcal{H}_{\lambda}$-module $M_{\lambda}$ is generated by $a_{1}$; it follows that in this case the image of $\mu_{\lambda}$ is the left ideal of $\mathcal{H}_{\lambda}$ generated by $X_{\lambda}$. From Theorem 0.2 it follows that there exists a finite subset $S_{0}$ of $\mathbf{C}^{*}$ such that $-1 \in S_{0}$ and such that:

(a) for $\lambda \in \mathbf{C}^{*}-S_{0}, \mu_{\lambda}: M_{\lambda} \rightarrow \mathcal{H}_{\lambda} X_{\lambda}$ is an isomorphism of $\mathcal{H}_{\lambda}$-modules. (Examples in small rank suggest that one can take $S_{0}=\{1,-1\}$.)

4.11. We now assume that $\lambda$ in 4.10 is such that $\lambda^{2}=q$ where $q$ is a power of a prime number. We write $\lambda=\sqrt{q}$. Let $G$ be a split semisimple algebraic group defined over the finite field $\mathbf{F}_{q}$ and let $G\left(\mathbf{F}_{q}\right)$ be the (finite) group of $\mathbf{F}_{q}$-rational points of $G$. Let $\mathcal{B}$ be the flag manifold of $G$ and let $\mathcal{B}\left(\mathbf{F}_{q}\right)$ be the set of $\mathbf{F}_{q}$-rational points of $G$. Let $\mathcal{F}$ be the vector space of functions $\mathcal{B}\left(\mathbf{F}_{q}\right) \rightarrow \mathbf{C}$. For any $B \in \mathcal{B}\left(\mathbf{F}_{q}\right)$ let $f_{B} \in \mathcal{F}$ be the function defined by $f_{B}\left(B^{\prime}\right)=\sqrt{q}^{l(w)}$ for any $B^{\prime} \in \mathcal{B}\left(\mathbf{F}_{q}\right)$ such that $\left(B, B^{\prime}\right)$ are in relative position $w \in W$. Let $\mathcal{F}^{\prime}$ be the $\mathbf{C}$-subspace of $\mathcal{F}$ spanned by the functions $f_{B}$ for various $B \in \mathcal{B}\left(\mathbf{F}_{q}\right)$. Note that $\mathcal{F}$ has a natural linear action of $G\left(\mathbf{F}_{q}\right)$ whose commuting algebra can be identified with $\mathcal{H}_{\sqrt{q}}$. Then $\mathcal{F}^{\prime}$ is a $G\left(\mathbf{F}_{q}\right)$-invariant space of $\mathcal{F}$. Moreover, we have $\mathcal{F}^{\prime}=X_{\sqrt{q}} \mathcal{F}$. For each two-sided cell $c$ of $W$ we denote by $\mathcal{F}_{c}$ (resp. $\left.\mathcal{F}_{c}^{\prime}\right)$ the sum of all simple $G\left(\mathbf{F}_{q}\right)$-submodules of $\mathcal{F}$ (resp. $\mathcal{F}^{\prime}$ ) which belong to $c$ in the classification of [L1]. Note that $\mathcal{F}_{c}$ is an $\mathcal{H}_{\sqrt{q}}$-submodule of $\mathcal{F}$ and that $\mathcal{F}_{c}^{\prime}=X_{\sqrt{q}} \mathcal{F}_{c}$. We have the following result.

Proposition 4.12. Assume that $\sqrt{q} \notin S_{0}$. Let $a^{\prime}=a\left(w_{0} w\right)$ where $w$ is any element of $c$ and $w_{0}$ is the longest element of $W$.

(a) We have $\operatorname{dim}\left(\mathcal{F}_{c}^{\prime}\right)=P_{c}(q)$ where $P_{c} \in \mathbf{N}[t]$ (t an indeterminate) is of the form $t^{a^{\prime}}+$ higher powers of $t$. Moreover, $P_{c}(1)$ is the number of involutions contained in c.

(b) We have $\operatorname{dim}\left(\mathcal{F}^{\prime}\right)=P(q)$ where $P \in \mathbf{N}[t]$ is such that $P(1)$ is the number of involutions in $W$.

We prove (a). We can assume that $W$ is irreducible. The simple $\mathcal{H}_{\sqrt{q}}$-modules which belong to $c$ can be indexed as in [L1] by a subset $I$ of $M(\Gamma)$ (see 3.1) for a certain finite group $\Gamma$ associated to $c$; we write $\epsilon_{i}$ for the simple $\mathcal{H}_{\sqrt{q}}$-module indexed by $i \in I$ and $\rho_{i}$ for the corresponding simple $G\left(\mathbf{F}_{q}\right)$-module appearing in $\mathcal{F}$. 
We apply 4.1 (a) with $A=\mathcal{H}_{\sqrt{q}}, \mathcal{X}=X_{\sqrt{q}}, E=\mathcal{F}_{c}$. We see that

$$
\operatorname{dim}\left(\mathcal{F}_{c}^{\prime}\right)=\operatorname{dim} \operatorname{Hom}_{\mathcal{H}_{\sqrt{q}}}\left(\mathcal{H}_{\sqrt{q}} X_{\sqrt{q}}, \mathcal{F}_{c}\right) .
$$

Using 4.10(a) we deduce

$$
\operatorname{dim}\left(\mathcal{F}_{c}^{\prime}\right)=\operatorname{dim} \operatorname{Hom}_{\mathcal{H}_{\sqrt{q}}}\left(M_{\sqrt{q}}, \mathcal{F}_{c}\right)=\sum_{i \in I}\left(\epsilon_{i}: M_{\sqrt{q}}\right) \operatorname{dim} \rho_{i}
$$

where $\left(\epsilon_{i}: M_{\sqrt{q}}\right)$ is the multiplicity of $\epsilon_{i}$ in $M_{\sqrt{q}}$. As explained in the remarks after Theorem 0.2 , the multiplicity $\left(\epsilon_{i}: M_{\sqrt{q}}\right)$ can be obtained from [Ko] namely,

if $|I|=2$, then $\left(\epsilon_{i}: M_{\sqrt{q}}\right)=1$ for $i \in I$;

if $|I| \neq 2$, and $i=(x, \rho) \in I$, then $\left(\epsilon_{i}: M_{\sqrt{q}}\right)$ is the multiplicity of $E_{x, \rho}$ in $\kappa$ (see 3.1 ) or equivalently, the multiplicity of $E_{x, \rho}$ in $V$ (see 3.2).

Thus, if $|I|=2$ we have

$$
\operatorname{dim}\left(\mathcal{F}_{c}^{\prime}\right)=\sum_{i \in I} \operatorname{dim} \rho_{i}
$$

if $|I| \neq 2$ we have

$$
\left.\operatorname{dim}\left(\mathcal{F}_{c}^{\prime}\right)=\sum_{(x, \rho) \in I} \text { (mult. of } E_{x, \rho} \text { in } V\right) \operatorname{dim} \rho_{(x, \rho)} .
$$

Let $d\left(\epsilon_{i}\right) \in \mathbf{N}[t]$ be the fake degree of $\epsilon_{i}$. If $|I|=2$ then by L1] we have $\sum_{i \in I} \operatorname{dim} \rho_{i}=\sum_{i \in I} \delta\left(\epsilon_{i}\right)$. If $|I| \neq 2$ then by [L1] we have $\operatorname{dim} \rho_{i}=\sum_{i^{\prime} \in I}\left\{i, i^{\prime}\right\} d\left(\epsilon_{i^{\prime}}\right)$ where $\left\{i, i^{\prime}\right\}$ is as in 3.3. Introducing this in (d) we obtain

$$
\left.\operatorname{dim}\left(\mathcal{F}_{c}^{\prime}\right)=\sum_{(x, \rho) \in I} \text { (mult. of } E_{x, \rho} \text { in } V\right) \sum_{(y, \sigma) \in I}\{(x, \rho),(y, \sigma)\} d\left(\epsilon_{y, \sigma}\right) .
$$

Using now 3.3(b) we obtain

$$
\operatorname{dim}\left(\mathcal{F}_{c}^{\prime}\right)=\sum_{(y, \sigma) \in I} d\left(\epsilon_{y, \sigma}\right)
$$

Here we have used the following two properties which are easily checked in each case.

$$
\text { (mult. of } \left.E_{x, \rho} \text { in } V\right) \neq 0 \Longrightarrow(x, \rho) \in I .
$$

If $(y, \sigma) \in I$, then the Frobenius-Schur indicator of $\sigma$ equals 1 .

We see that (e) holds both when $|I| \neq 2$ and when $|I|=2$. Now the first assertion of (a) follows immediately from (e); the second assertion of (a) also follows from (a) using the fact that $\left.d\left(\epsilon_{y, s}\right)\right|_{q=1}=\operatorname{dim}\left(\epsilon_{y, s}\right)$ and that $\sum_{(y, \sigma) \in I} \operatorname{dim}\left(\epsilon_{y, \sigma}\right)$ is equal to the number of involutions in $c$; see [Ge].

Clearly, (b) is a consequence of (a). The proposition is proved.

4.13. The proof of 4.12 shows that $\operatorname{dim}\left(\mathcal{F}^{\prime}\right)$ is equal to the sum of the fake degrees $d(\epsilon)$ of the various irreducible representations $\epsilon$ of $\mathcal{H}_{\sqrt{q}}$ (each one taken once).

\section{REFERENCES}

[Ge] M. Geck, Kazhdan-Lusztig cells and the Frobenius-Schur indicator, J. Algebra 398 (2014), 329-342, DOI 10.1016/j.jalgebra.2013.01.019. MR.3123768

[HZ] J. Hu and J. Zhang, On involutions in symmetric groups and a conjecture of Lusztig, Adv. Math. 287 (2016), 1-30, DOI 10.1016/j.aim.2015.10.003. MR3422674

[Ko] R. E. Kottwitz, Involutions in Weyl groups, Represent. Theory 4 (2000), 1-15 (electronic), DOI 10.1090/S1088-4165-00-00050-9. MR1740177 
[L1] G. Lusztig, Characters of reductive groups over a finite field, Annals of Mathematics Studies, vol. 107, Princeton University Press, Princeton, NJ, 1984. MR.742472

[L2] G. Lusztig, Leading coefficients of character values of Hecke algebras, The Arcata Conference on Representations of Finite Groups (Arcata, Calif., 1986), Proc. Sympos. Pure Math., vol. 47, Amer. Math. Soc., Providence, RI, 1987, pp. 235-262. MR.933415

[L3] G. Lusztig, Hecke algebras with unequal parameters, CRM Monograph Series, vol. 18, American Mathematical Society, Providence, RI, 2003. MR 1974442

[L4] G. Lusztig, Asymptotic Hecke algebras and involutions, Perspectives in representation theory, Contemp. Math., vol. 610, Amer. Math. Soc., Providence, RI, 2014, pp. 267-278, DOI 10.1090/conm/610/12156. MR.3220630

[L5] G. Lusztig, A bar operator for involutions in a Coxeter group, Bull. Inst. Math. Acad. Sin. (N.S.) 7 (2012), no. 3, 355-404. MR3051318

[L6] G. Lusztig, Some power series involving involutions in Coxeter groups, Represent. Theory 19 (2015), 281-289, DOI 10.1090/ert/472. MR 3418645

[LV] G. Lusztig and D. A. Vogan Jr., Hecke algebras and involutions in Weyl groups, Bull. Inst. Math. Acad. Sin. (N.S.) 7 (2012), no. 3, 323-354. MR 3051317

Department of Mathematics, Massachusetts Institute of Technology, Cambridge, MassachusetTs 02139

E-mail address: gyuri@math.mit.edu 